\title{
Representaciones léxicas: procesamiento y repercusión sobre el conocimiento bilingüe
}

\author{
Carlos Inchaurralde Besga
}

Universidad de Zaragoza

\subsection{Resumen}

El vocabulario se usa normalmente en la comunicación humana asociado a un conocimiento de tipo enciclopédico, y diferentes autores cognitivos nos ofrecen descripciones de cómo esta información enciclopédica almacenada en nuestra mente se puede utilizar y vincular a unidades léxicas concretas. Desde este punto de vista las unidades léxicas son simplemente puntos de acceso, y que, por lo tanto "los conceptos son simplemente rutinas". Este es el tipo de descripción que también proponen los psicólogos y los expertos en computación, con modelos en los que las redes semánticas y la activación de nodos son presupuestos importantes que explican el vínculo dinámico entre las palabras y el conocimiento enciclopédico. La construcción dinámica de las estructuras de conocimiento y los conceptos individuales asociados, así como las teorías de esquemas, según las cuales todo lo que sabemos del mundo está almacenado en grandes estructuras mentales, conforman un modelo de conocimiento que puede ser aplicado en otras áreas de estudio. Este modelo de conocimiento es incluso más interesante si examinamos la manera en que se utiliza en personas bilingües. La investigación actual nos sugiere que la memoria bilingüe está organizada como un único lexicón distribuido y no como dos lexicones accesibles por separado correspondientes cada uno a cada una de las dos lenguas; y, en este sentido, es posible analizar cómo la evidencia neurolinguística puede ayudarnos en el modelado del conocimiento multilingüe y su aplicación práctica para el tratamiento de la información.

Palabras clave: Representación léxicas. Organización del conocimiento. Representación del conocimiento. Lexicología. Semántica.

\subsection{Abstract}

Vocabulary in human communication is normally used in association with encyclopedic knowledge. Descriptions of how this encyclopedic information deep in our minds can be used and linked to single words appear in the work of different cognitive authors. From this point of view lexical units are merely points of access, and that therefore "concepts are simply entrenched cognitive routines". 
This is the kind of description proposed by psychologists and computer scientists, with models that involve semantic networking and activation as important principles explaining the link between words and encyclopedic knowledge dynamically. The suggestion that knowledge structures and the individual concepts associated with them are dynamically constructed together with "schemata" theories according to which everything we know about the world is stored in large mental knowledge structures, make up an interesting knowledge model that can be applied in other fields. This knowledge model involved in human communication is even more interesting if we examine it as it is used in bilinguals. Current research suggests that bilingual memory is organized as a single distributed lexicon rather than as two separately accessible lexicons corresponding to each language. In this respect, it will be analyzed here how neurolinguistic evidence can throw some light onto the modeling of multilingual knowledge in other domains.

Keywords: Lexical representation. Knowledge organization. Knowledge representation. Lexicology. Semantics.

\section{Introducción. Las palabras como entidades psicológicas}

Actualmente existe un debate importante en la escena lingüística sobre cómo abordar el significado léxico. Por un lado, tenemos la perspectiva más tradicional, según la cual tiene que ser posible describir en una entrada de diccionario la parte más importante de la información semántica y gramatical asociada a una palabra. Esta es la perspectiva guiada por la "metáfora del conducto" (Reddy, 1979), "que ve las unidades léxicas como contenedores para el significado" (Langacker, 1987, p. 162). Frente a ella, tenemos la perspectiva "enciclopédica", para la que el significado de las palabras no puede considerarse aisladamente, sino conectado con todo un gran conocimiento del mundo. Esta última concepción concuerda con la evidencia neurolingüística disponible, y está comúnmente aceptada entre los lingüistas cognitivos.

Sin embargo, hay que reconocer, en primer lugar, que la metáfora del conducto tiene cierta base, tanto desde un punto de vista intuitivo como científico. Las palabras pueden ser procesadas individualmente, y muchas características de su comportamiento psicolingüístico son fácilmente explicables si nos planteamos la existencia de una lista mental ordenada de entradas léxicas. En la literatura científica podemos encontrar varios fenómenos que se suelen dar en los experimentos relacionados con el uso del vocabulario: las palabras más frecuentes suelen identificarse antes y recordarse más fácilmente, las pseudo-palabras que se parecen fonética o gráficamente a palabras reales suelen tardar más en ser rechazadas que otras palabras no válidas, ciertas palabras-estímulo tienen influencia en los tiempos de reacción asociados a otras palabras, y los casos en los que aparece alguna forma de ambigüedad léxica son abundantes (como mencio- 
namos más detalladamente en Inchaurralde, 2000, p. 99). Respecto a esta última cuestión, la de la diversidad de significados, desde un punto de vista neurolingüístico, se ha planteado un interrogante interesante: ¿Activamos todos los significados de una palabra cuando hay ambigüedad, o activamos sólo un significado guiados por el contexto? De acuerdo a los modelos llamados interactivos (Morton, 1979; Patterson y Shewell, 1987), cada palabra aparece representada en el lexicón por un dispositivo que es sensible a ciertos tipos de información, y esta información proviene tanto del canal acústico-sensorial como del contexto lingüístico. El reconocimiento de las unidades léxicas adopta la forma de una competición, en la que las diferentes entradas son expuestas simultáneamente a una activación acumulativa, hasta que la cantidad de activación de una de ellas sobrepasa un determinado límite y es elegida. Frente a este enfoque tenemos los modelos autónomos, en los que se lleva a cabo un proceso de búsqueda exhaustiva en una lista mental. Forster $(1976,1990)$ nos dice que la identificación léxica se lleva a cabo en dos etapas: primero se compara la representación perceptiva del estímulo con su representación fonológica o gráfica. Una vez se ha encontrado la palabra, una segunda etapa comprende la búsqueda de información semántica y sintáctica en una gran base de datos mental.

Tanto el enfoque interactivo como el autónomo permiten explicar los fenómenos reseñados más arriba. Los efectos de frecuencia pueden ser explicados por los modelos interactivos en términos de los diferentes niveles de activación que pueden alcanzar las unidades léxicas. Los modelos autónomos, en cambio, los explican por el hecho de que las palabras más frecuentes aparecen las primeras en las listas mentales. Los efectos de facilitación con otros estímulos se pueden explicar mediante la interacción, la cual también se puede dar con posterioridad al acceso a la lista mental postulado por los modelos autónomos. El tiempo invertido en rechazar pseudo-palabras se puede explicar en términos del tiempo que se tarda en hacer una búsqueda exhaustiva en las listas mentales.

Como consecuencia, nos hemos encontrado también con modelos producto de la combinación de estos dos puntos de vista (cf. Marslen-Wilson, 1987); pero, en cualquier caso, lo que a nosotros nos interesa resaltar es que las palabras son claramente identificadas como unidades y, por lo tanto, es explicable que exista una concepción de la información léxica como algo "contenido", "guardado" en las palabras, dando apoyo a la metáfora del conducto.

\section{Tipos de significado}

Sin embargo, esa información "contenida" en las palabras tiene muchos formatos diferentes, y puede llegar a ser realmente compleja. Normalmente, los lingüistas han distinguido entre denotación y connotación. La denotación tiene que ver con la "referencia" al mundo, y constituye un tipo de significado "objetivo".

Scire. 8: 1 (en.-jun. 2002) 41-54. 
La connotación, sin embargo, se refiere a un tipo de significado "subjetivo", relacionado con el contenido evaluativo de las palabras.

El significado denotativo está ligado a un conocimiento que está almacenado de algún modo en nuestra mente. La psicología cognitiva asume a este respecto que hay distintos tipos de almacenamiento en nuestra memoria. Como es bien conocido, toda la información permanente está almacenada en la memoria a largo plazo, por oposición a la memoria a corto plazo, que sólo almacena información útil durante un corto espacio de tiempo (Tulving, 1979). En la memoria a largo plazo tenemos dos tipos distintos: memoria episódica (relacionada con situaciones concretas en las que el sujeto ha participado) y memoria semántica (memoria no relacionada con ninguna situación concreta, que almacena el conocimiento general sobre todo lo que ocurre en el mundo). A través de la interacción con la realidad, los niños aprenden el vocabulario funcionalmente, en su uso dentro de situaciones concretas (cf. Nelson, 1985). Esto hace que la memoria episódica vaya almacenando información que servirá para ir construyendo las redes de conocimiento presentes en la memoria semántica. De hecho, cuanta más exposición tenga el sujeto a las diferentes manifestaciones del significado denotativo de una palabra, más claramente quedará establecido en sus representaciones mentales. Una distinción relevante a este respecto es la que planteamos en Inchaurralde (1989) al mencionar los conceptos de extensión cognitiva e intensión cognitiva. La extensión cognitiva hace alusión precisamente a esta adquisición del significado a través de la experiencia. Que el significado se va creando dinámicamente en nuestra interacción con el mundo es algo que está apoyado en cierta medida por trabajos como los de Barsalou y colaboradores (Barsalou, 1982, 1993; Barsalou y Sewell, 1984; Barsalou y Prinz, 1997). La intensión cognitiva, en cambio, hace referencia a la manera en que se adquiere el vocabulario en la instrucción formal, a través de definiciones, que resumen de manera condensada el significado del término. Ésta es la manera en que se adquiere normalmente el significado del vocabulario relacionado con disciplinas muy especializadas. El vocabulario de uso corriente, sin embargo, se adquiere normalmente a través de la interacción con el medio, de manera extensional.

Los enfoques tradicionales del significado léxico han sido intensionales, basados en un modelo de rasgos suficientes y necesarios. Este era el tratamiento característico de los modelos estructuralistas y el análisis componencial (cf. Nida, 1975). Pero tras la "teoría de prototipos" elaborada por la psicóloga Eleanor Rosch y sus colaboradores (Rosch, 1973, 1975, 1977, 1978, 1981; Rosch y Lloyd, 1978; Rosch y Mervis, 1975), la perspectiva de la lingüística contemporánea sobre el léxico ha cambiado radicalmente (véase Taylor, 1989; Geeraerts, 1993, 1997; etc.). El significado ya no está formado por rasgos discretos, suficientes y necesarios (cf. Geeraerts, 1988), sino que tiene una estructura

Scire. $8: 1$ (en.-jun. 2002) 41-54. 
más difusa. Sin embargo, esto no quiere decir que el vocabulario no esté estructurado. Muy al contrario, presenta una estructura característica. Alrededor de un significado central gravita toda una constelación de diferentes significados relacionados, en muchos casos originados a través de procedimientos de extensión del significado, que pueden ser metonímicos (por ejemplo, cabeza en "Tiene buena cabeza", refiriéndose a dotes intelectuales), metafóricos (por ejemplo, pie en "Al pie de la montaña"), o basados en procesos de generalización (por ejemplo, escuela como corriente de pensamiento frente a escuela como institución de aprendizaje) o de especialización (por ejemplo, escuela para referirse a un curso especial). Todos los significados así relacionados pueden constituir lo que se ha llamado una red radial (Fig. 1).

Además de redes radiales también tenemos otros tipos de configuraciones. Cruse (1986) menciona cómo hay ciertas estructuras de significado que plantean problemas al concebirse como redes. Este es el caso con los espectros de sentidos (Cruse, 1986: 73), en los que hay una relación entre significados contiguos, pero no entre los extremos de una serie de significados relacionados. Asimismo, las redes semánticas se pueden representar mediante intersecciones de conjuntos de características, presentando relaciones "de parecido familiar" (family resemblance).

Esta manera de concebir el significado, no basándose en una acumulación de unos determinados rasgos semánticos, sino basándose en la relación que se establece entre el sentido de unos términos y otros, y estableciendo una estructura difusa, de niveles de "prototipicidad", muestra que el significado no está estructurado en "cajas" de contenido (la metáfora del conducto), sino más bien repartido en diferentes direcciones dentro de las unidades léxicas. El significado tiene, por lo tanto, una "naturaleza distribuida".

La naturaleza distribuida del significado no sólo comprende conocimiento

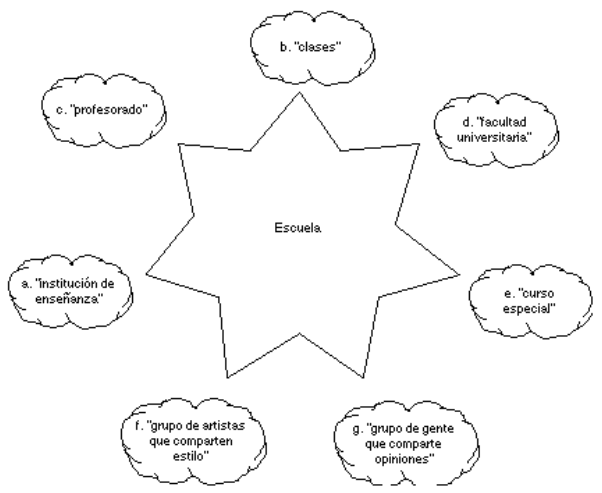

Fig. 1. Red radial de los sentidos de la palabra escuela (Inchaurralde y Vázquez, 2000, 32).

Scire. $8: 1$ (en.-jun. 2002) 41-54. 
"objetivo" sobre el mundo, denotativo, sino que también es aplicable a valores connotativos. Osgood (1976) probó que la evaluación en términos de bueno/malo es posiblemente el factor más importante en la connotación de muchas palabras. Para este autor, el significado evaluativo que asignamos a las cosas es equivalente a la actitud individual que se pone de manifiesto mediante la elección léxica, y dicha actitud puede medirse. En sus experimentos, los sujetos experimentales tenían que asignar valores a una muestra de vocabulario mediante el uso de diferentes escalas con adjetivos. Tras hacer un análisis factorial de los resultados, Osgood pudo inferir que había tres dimensiones principales: evaluación, potencia, y actividad. El procedimiento de factorización usado por este autor revelaba también otras dimensiones, pero sus valores eran muy bajos para ser consideradas significativas. En cualquier caso, la dimensión evaluativa, la que asignaba valores en términos de "bueno" o "malo", era la que más destacaba frente a las demás. De aquí podemos deducir que dicha dimensión es un elemento fundamental del valor connotativo de las palabras. Junto a este tipo de significado connotativo tenemos conocimiento de índole cultural, sobre determinados valores culturales asociados a determinadas palabras, que son, por tanto, difíciles de traducir (cf. el concepto de palabra clave cultural en Wierzbicka, 1997). Este conocimiento de índole cultural puede llegar también a incorporar multitud de elementos denotativos (cf. el concepto de guión léxico cultural en Inchaurralde, 1994). En cualquier caso, todos estos tipos de significados no parecen estar configurados de acuerdo a rasgos suficientes y necesarios, sino de acuerdo a una naturaleza distribuida característica del significado léxico.

En la lingüística cognitiva se asume que el carácter enciclopédico del significado léxico obedece al funcionamiento cognitivo peculiar de las unidades léxicas. Según Langacker (1987, p. 162), la unidades léxicas son simplemente puntos de acceso a redes cognitivas, y, por lo tanto, los conceptos no son sino rutinas cognitivas que desencadenan una secuencia de activación dentro de la red. Claramente, el modelo de representación que se está sugiriendo aquí es el que nos proporciona el conexionismo, que simula el funcionamiento mental humano a partir de redes neuronales artificiales, y que busca reproducir en estas simulaciones las características peculiares de este funcionamiento, frente a otros modelos que simplemente quieren aislar los grandes procesos involucrados, fácilmente identificables, con representaciones más simples (por ejemplo, los diagramas de flujo usados por los psicólogos cognitivos de los años 70).

Una mirada a la manera en que funciona un modelo de estas características nos permite comprender mejor esta concepción del significado léxico. Por ejemplo, Anderson (1983) nos presenta el modelo ACT. En este modelo se distinguen distintos tipos de memoria: declarativa, de producción, y de trabajo. Para poder recuperar información de la memoria declarativa y así poder usarla en la memoria de trabajo, hace falta cierta cantidad de energía en forma de activación. Hay

Scire. $8: 1$ (en.-jun. 2002) 41-54. 
tres formas de activación: percepción, activación en progreso (spreading activation), y focalización. Cuando oímos una palabra o vemos un objeto se puede activar un nodo de acceso a la red. Esto es lo que se llama percepción. La activación en progreso se refiere a lo que ocurre cuando un concepto activo extiende su actividad a conceptos relacionados de la red, y se puede dar en forma convergente (cuando un concepto es activado simultáneamente por muchos otros) o en forma divergente (cuando hay una gran competición por la activación de los diferentes nodos) (cf. Deane, 1992, p. 35). La focalización se refiere a la activación consciente y forzada de un concepto que no recibe suficiente activación de otro tipo. Es precisamente la activación lo que permite la conexión entre unidades léxicas y nuestro conocimiento del mundo, enciclopédico, de una manera dinámica.

\section{El vocabulario especializado}

Dentro de la problemática del conocimiento vinculado a las unidades léxicas tenemos que mencionar también el problema de la delimitación de niveles diferentes de conocimiento experto en dominios concretos de la misma lengua o en dos lenguas diferentes, o lo que se ha llamado la distinción entre "categorías del lego" (folk categories) y "categorías del experto" (expert categories) (cf. Taylor, 1989: 72). Esta distinción tiene que ver con la noción de Putnam de división de labor dentro de una comunidad de habla (1975). Los hablantes normales identifican los objetos del mundo a través de "estereotipos", que están basados en nuestro conocimiento de atributos de percepción e interacción presentes en determinados casos. Al mismo tiempo, hay grupos de expertos para los que los mismos objetos o nociones se definen de una manera más científica y experta. Tenemos el ejemplo bien conocido del término "ballena" en taxonomías populares frente a "ballena" en una taxonomía biológica científica. Una ballena es un tipo de "pescado" en una taxonomía popular, mientras que es siempre un mamífero en biología. Hoy en día toda persona alfabetizada sabe que una ballena es un mamífero, aunque puede tener un estatus de pez en expresiones idiomáticas y en usos populares del término. Esta cuestión es incluso más relevante para el uso de ciertos términos en dominios especializados, pues puede darse el caso de que los usuarios de la lengua no sean conscientes del significado especializado a no ser que se les haga notar explícitamente. La importancia de un lenguaje preciso en la ciencia, el derecho y otras disciplinas otorga un papel muy importante a las definiciones. En el campo científico, cualquier discusión necesita una definición clara de los términos usados, pues si no es así, los científicos pueden acabar discutiendo cosas diferentes. En el derecho, los términos que son importantes para ejecutar determinadas leyes necesitan ser definidos claramente, dado que en otro caso dichas leyes tendrían muchas interpretaciones, y las disputas serían difíciles de resolver.

En muchos casos, existen términos cargados de un significado cultural que es

Scire. 8: 1 (en.-jun. 2002) 41-54. 
de difícil traducción. Cuando estos términos conciernen áreas especializadas, se preserva el término, aunque muchas veces se intente adaptar su pronunciación a la de la nueva lengua que los adopta. Un campo con un gran grado de especialización, a pesar de ser un área de experiencia cotidiana, es el de la cocina. Aquí podemos encontrar unidades léxicas como "espagueti", "quiche lorraine", "sushi" y muchas otras que se han originado en otras lenguas y no tienen traducción posible, puesto que corresponden a un área muy especializada y condicionada culturalmente. Este es un ejemplo de especialización en varios idiomas simultáneamente, pero encontramos dominios que encuentran una mayor especialización en unas lenguas que en otras. En estos casos, de Beaugrande (1997, p. 326) nos recuerda que "Un LFE [Lenguaje para Fines Específicos] puede estar influido por otros lenguajes además del LPG [Lenguaje de Propósito General] en el que se basa y puede llegar a convertirse en una 'interlengua' o un modo de 'cambio de código"” (...) "Este factor ayuda a potenciar la influencia mundial de los términos ingleses, franceses y alemanes en LFEs obtenidos a partir de otros LGPs". Es lo que ocurre, por ejemplo, en el área de la informática, donde la mayoría de la investigación, comunicación y mercadotecnia internacional en este campo especializado se llevan a cabo mediante el uso del inglés como idioma instrumental. En español, como en muchas otras lenguas, unidades léxicas como "hardware" y "software" se usan constantemente, aun cuando nadie esté seguro de cómo se pronuncian, y lo mismo ocurre con incontables términos dentro de este dominio. A veces es posible utilizar términos equivalentes en la lengua meta, pero la tendencia general es utilizar el término inglés, con sus connotaciones de excelencia técnica y sugerencias de conocimiento "experto" del mundo. De la misma manera, otros dominios, como la economía, el comercio, la tecnología y áreas científicas en general, están llenos de expresiones inglesas. La palabra "marketing" es hoy en día un término que todo el mundo reconoce en español, y su alternativa, "mercadotecnia", se ha convertido en un término en peligro de extinción. "Marketing", además, ha perdido su condición de unidad léxica de un dominio experto, pues ha pasado al lenguaje general. Frente a esto, existen otros términos que se usan dentro de un ámbito especializado muy limitado (por ejemplo, el uso de "outsourcing" en la jerga empresarial).

Las palabras extranjeras de dominios especializados normalmente sufren cierta adaptación fonética, y a veces gráfica, a las peculiaridades formales de la lengua que las recibe. Esto es incluso más claro cuando el dominio especializado es todo un dominio cultural. De este fenómeno es un claro ejemplo el japonés, una lengua con cinco vocales, como el español, con limitaciones en el uso de sílabas cerradas (sólo aquellas acabadas en "n") y con un número reducido de consonantes. En esta lengua son muy corrientes los préstamos adaptados de la lengua inglesa. Tenemos, por ejemplo, "juusu" por juice (zumo), "koohi" por coffee (café), "fooku" por fork (tenedor), "sakkaa" por soccer (fútbol), etc. Pero en estos 
casos, los nuevos términos se incorporan al lenguaje. A veces, una disciplina concreta es tan dependiente de una lengua dada que no basta con incorporar parte del léxico a la nueva lengua. Pensemos, por ejemplo, en la informática, donde la velocidad a la que se mueve la disciplina, y su carácter tan dinámico, hacen que sea recomendable aprenderla y manejarla en su idioma más usual, que es el inglés. Esto nos lleva a la consideración de distintos tipos de conocimiento asociados a diferentes lenguas. Para un profesor de idiomas, esta asociación posibilita un soporte adecuado para el aprendizaje de una nueva lengua. Precisamente, los enfoques basados en tareas (Long, 1985; Prabhu, 1987; Long y Crookes, 1992) ponen énfasis en el hecho de que llevando a cabo tareas de cierta complejidad intelectual con la nueva lengua se interioriza mejor el sistema lingüístico, dado que hay un desplazamiento del interés por la forma a un interés por el contenido, y la forma en que se expresa dicho contenido se va creando de una manera inductiva, hasta obtener un grado muy alto de dominio de la lengua en aprendizaje. Sin embargo, nuestra línea principal de argumentación va en la otra dirección: conocer la forma ayuda a utilizar el nuevo contenido. En cualquier caso, se puede considerar que ambas direcciones, la del contenido a la forma, y la de la forma al contenido, operan simultáneamente, y que no es tan importante la problemática de la direccionalidad de esta secuencia como la manera en que varias lenguas y varios tipos de conocimiento asociados a ellas pueden coexistir en forma de una gran red semántica común.

\section{El conocimiento bilingüe}

Precisamente, una cuestión importante aquí es si debemos considerar redes separadas o una única red. La evidencia parece demostrar que el modelo adecuado es una única red, pues se dan muchísimos fenómenos experimentales que muestran interferencia entre las distintas lenguas que pueden coexistir en la mente de una persona. Muchos experimentos han mostrado el papel de la interferencia entre lenguas, aun en los casos en que los sujetos bilingües han sido puestos en contextos monolingües (De Groot y Nas, 1991; Beauvillain, 1992; Keatly y de Gelder, 1992; Grainger y O'Regan, 1992; Keatley, Spinks y De Gelder, 1994; French y Ohnesorge, 1996, 1997; Fox, 1996). Se puede intentar explicar la interferencia a partir de modelos de activación interactiva como el de McClelland y Rumelhart (1981). Sin embargo, este tipo de modelos, aunque están basados en el paradigma conexionista, no son apropiados para aprender. French (1998) nos demuestra cómo una arquitectura conexionista distribuida (a diferencia del modelo de McClelland y Rumelhart, localista) capaz de aprender permite reproducir efectos de interferencia similares a los observados con sujetos reales. French (1998, p. 368) utiliza una red conexionista recurrente simple —-simple recurrent connectionist network (SRN) — basada en la propuesta de Elman (1990) para cre-

Scire. 8: 1 (en.-jun. 2002) 41-54. 
ar un entorno de simulación apropiado. Este modelo de red mostrará ciertas peculiaridades (desarrolla progresivamente representaciones ocultas que agrupan formas gramaticales y diferentes lenguas, presenta efectos de interferencia entre lenguas, es muy resistente a las lesiones, y la desorganización interna que presenta en algunos casos es causada sólo por la lesión de algunos nodos muy concretos) que coinciden con el comportamiento léxico observado en sujetos bilingües.

El experimento planteado por French (1998) se basa en dos micro-lenguajes artificiales llamados Alpha y Beta. Alpha está formado por subject nouns - como boy, girl, man, woman - verbs — lifts, touches, sees, pushes - y object nouns toy, ball, book, pen. Beta, por su parte, se compone también de subject nouns garcon, fille, homme, femme-, verbs - souleve, touche, voit, pousse- y object nouns - jouet, ballon, livre, stylo- (French, 1998: 369). Con el vocabulario de estos dos micro-lenguajes se forman oraciones siguiendo la estructura gramatical SVO (NOMBRESUJETO - VERBO - NOMBREOBJETO). Un programa genera secuencias de oraciones permitidas, y aleatoriamente va cambiando de microlenguaje (siempre que la oración anterior haya quedado completa). El texto obtenido se va suministrando a la red neuronal, que aprende a formar agrupaciones por lenguajes y por categorías gramaticales después de haber recibido 20000 oraciones (60000 palabras). Tras el período de aprendizaje, French observa que el daño a distintos nodos de la red no afecta a su comportamiento global. Esto es similar a lo que ocurre con el trauma cerebral en los hablantes bilingües: en general, no da como resultado la pérdida de la capacidad en una lengua o la mezcla de distintas lenguas. Sin embargo, sí que se dan algunos casos concretos, muy raros, en los que estos efectos se pueden producir. En la simulación de French (1998), este fenómeno también se daba en algún caso concreto tras quitar un nodo de la red.

Otro fenómeno observado en el que el modelo real y el modelo simulado coinciden es la disminución de la facilitación (priming) homográfica (French, 1998: 371). Con homógrafos interlingüísticos (por ejemplo, fin es "fin" en francés y "aleta" en inglés) aparece mucha menos facilitación en entornos bilingües que en entornos monolingües. Este tipo de situación se intentó emular con la red neuronal usada por French, y se observaron efectos parecidos al utilizar pseudo-palabras difíciles de clasificar como pertenecientes a Alpha o a Beta. (French, 1998: 371-372). Frente a la cuestión de si el modelo planteado en este experimento puede ser ampliable y generalizable a vocabularios más amplios, French (French, 1998: 372) argumenta que ha sido probado con 1536 palabras (256 por categoría) utilizando la misma gramática simple de tipo SVO. Los resultados son similares al experimento con un vocabulario más reducido. Aparentemente, no hay razón por la que no se pudieran dar los mismos resultados con vocabularios muchísimos más grandes. Parece que sí tiene cierto grado posible de generalización, lo que permite utilizarlo para apoyar nuestro argumento de que no necesitamos dos redes

Scire. $8: 1$ (en.-jun. 2002) 41-54. 
para representar un vocabulario bilingüe o, más concretamente, el modelo de activación interactiva bilingüe mencionado por Grainger (1993). Realmente, este modelo conexionista basado en una red neuronal distribuida puede reproducir bastante bien determinado comportamiento de una mente que "piensa" en dos idiomas, lo que equivale a sugerir que el almacenamiento y utilización de un conocimiento bilingüe es posible de esta manera; y una gran red neuronal debería permitirnos, con el entrenamiento adecuado, registrar conocimiento multidisciplinar en dos o más lenguas. El problema de la identificación del vocabulario se puede plantear fácilmente a partir de una intersección de características que lo definen, a modo de rasgos, de acuerdo a la concepción tradicional del significado léxico. La vinculación conceptual entre términos, por otra parte, quedaría establecida por la propia red, y sería la propia red, internamente, y de manera opaca al usuario externo, la que mantendría conexiones apropiadas entre los nodos.

\section{Conclusión}

El significado léxico dista de tener una modalidad única de representación. Como hemos visto, puede tener muchas maneras diferentes de configurarse. En este sentido, la mejor estrategia de representación es la más flexible, dando cabida a fenómenos de prototipicidad, semejanzas de sentidos, relaciones configuradas en forma de redes radiales, conexiones con conocimiento general sobre el mundo o con conocimiento de tipo cultural, contenido evaluativo, distintos registros especializados, etc. La existencia de todos estos fenómenos y el hecho innegable de que el conocimiento y, por tanto, la información asociada a las unidades léxicas, tiene una naturaleza distribuida hace que sea importante la consideración del paradigma conexionista como el modelo adecuado para dar cuenta de estos fenómenos. A todo esto podemos añadir, como un elemento que aparentemente complica más la situación, la posibilidad de vinculación de determinado vocabulario o de determinados usos del vocabulario a distintos idiomas. A pesar de que cierta evidencia nos ha mostrado en el pasado la localización en distintas áreas cerebrales del vocabulario de lenguas distintas, la información disponible ahora nos hace ver que también al utilizar vocabulario bilingüe o multilingüe la información puede estar muy distribuida. En este sentido, hemos hecho referencia a un experimento de simulación que muestra de qué manera una red neuronal puede emular este comportamiento. Tenemos así que una red única para conocimiento bilingüe aparece como un modelo de representación útil para almacenamiento y recuperación de la información asociada, y este hecho debe ser tenido en cuenta con el fin de utilizarlo en aplicaciones prácticas.

\section{Referencias}

Anderson, J. R. (1983). The architecture of cognition. Cambridge : Harvard Univ. Press, 1983. Barsalou, Lawrence W. (1982). Context-independent and context-dependent information

Scire. 8: 1 (en.-jun. 2002) 41-54. 
in concepts. // Memory and Cognition. 10 (1982) 82-93.

Barsalou, Lawrence W. (1993) Flexibility, structure and linguistic vagary in concepts. Manifestations of a compositional system of perceptual symbols. // Collins, Alan F. ; Gathercole, Susan E. ; Conway, Martin A. ; Morris, Peter E. (eds.) Theories of memory. Hillsdale : Lawrence Erlbaum, 1993. 29-101.

Barsalou, Lawrence W. ; Sewell, Daniel R. (1984). Constructing representations of categories from different points of view. // Emory Cognition Project Technical Report 2. Atlanta : Emory University, 1984.

Barsalou, Lawrence W. (1993). Flexibility, structure and linguistic vagary in concepts. Manifestations of a compositional system of perceptual symbols. // Collins, Alan F. ; Gathercole, Susan E. ; Conway, Martin A. ; Morris, Peter E. (eds.). Theories of Memory. Hillsdale : Lawrence Erlbaum, 1993. 29-101.

Barsalou, Lawrence W. ; Prinz, Jesse J. (1997). Mundane creativity in perceptual symbol systems. // Ward, Thomas B. ; Smith, Steven M. ; Vaid, Jyotsna (eds.). Creative thought: An investigation of conceptual structures and processes. Washington, D.C. : American Psychological Association, 1997. 267-307

Beaugrande, Robert de (1997). New foundations for a science of text and discourse: cognition, communication, and the freedom of access to knowledge and society. Norwood, New Jersey : Ablex Publishing Corporation, 1997.

Beauvillain, C. (1992). Orthographic and lexical constraints in bilingual word recognition. // Harris, R. (ed.) Cognitive processing in bilinguals. Amsterdam : Elsevier Science Publishers, 1992. 221-235.

Cruse, D. Alan (1986). Lexical semantics. Cambridge : Cambridge University Press, 1986.

De Groot, A. ; Nas, G. (1991). Lexical representation of cognates and noncognates in compound bilinguals. // Journal of Memory and Language. 26 (1991) 658-672.

Deane, Paul D. (1992). Grammar in brain and mind. Explorations in cognitive syntax. Berlin : Mouton de Gruyter, 1992.

Elman, J. (1990). Finding structure in time. // Cognitive Science. 14 (1990) 179-211.

Fillmore, Charles J. (1982). Frame semantics. // Linguistic Society of Korea (ed.) Linguistics in the morning calm. Seoul : Hanshin, 1982. 111-137.

Forster, Kenneth I. (1976). Accessing the mental lexicon. // Wales, Roger J. ; Walker, Edward C. T. (eds.). New approaches to language mechanisms. Amsterdam: NorthHolland, 1976. 257-287.

Forster, Kenneth I. (1990). Lexical processing. // Osherson, Daniel N. ; Lasnik, Howard (eds). Language: An invitation to cognitive science : vol. 1. Cambridge: BradfordM.I.T. Press, 1990. 95-131.

Fox, E. (1996). Cross-language priming from ignored words: Evidence for a common representational system in bilinguals. // Journal of Memory and Language. 35 (1996) 353-370.

French, Robert M. (1998). A simple recurrent network model of bilingual memory. // Proceedings of the Twentieth Annual Cognitive Science Society Conference. New Jersey : LEA, 1998. 368-373.

French, Robert M. ; Ohnesorge, C. (1996). Using interlexical nonwords to support an interactive-activation model of bilingual memory. // Proceedings of the Eighteenth

Scire. $8: 1$ (en.-jun. 2002) 41-54. 
Annual Cognitive Science Society Conference. New Jersey : LEA, 1996. 318-323.

French, Robert M. ; Ohnesorge, C. (1997). Homographic self-inhibition and the disappearance of priming: More evidence for an interactive-activation model of bilingual memory. // Proceedings of the Nineteenth Annual Cognitive Science Society Conference. New Jersey : LEA, 1997. 241-246.

Geeraerts, Dirk (1988). On necessary and sufficient conditions. // Journal of Semantics. 5 (1988) 275-291.

Geeraerts, Dirk (1993). Vagueness's puzzles, polysemy's vagaries. // Cognitive Linguistics. 4 (1993) 223-272.

Geeraerts, Dirk (1997). Diachronic prototype semantics: a contribution to historical lexicology. Oxford: Clarendon Press, 1997.

Grainger, J. ; O'Regan, J. (1992). A psychophysical investigation of language priming effects in two English-French bilinguals. // European Journal of Cognitive Psychology. 4 : 4 (1992) 323-339.

Grainger, J. (1993) Visual recognition in bilinguals. // Schreuder, R. ; Weltens, B. (eds.). The bilingual lexicon. Amsterdam : John Benjamins, 1993.

Inchaurralde, Carlos (1989). Hacia un modelo integral de representación semántica: Algunas observaciones. // Martín Vide, Carlos (ed.). Actas del V Congreso de Lenguajes Naturales y Lenguajes Formales. Barcelona : P.P.U., 1989. 587-594.

Inchaurralde, Carlos (1997). What is behind a word: Cultural scripts. // Pütz, Martin (ed.). The cultural context in foreign language teaching. Frankfurt: Peter Lang, 1997. 55-66.

Inchaurralde, Carlos (2000). Lexicopedia. // Peeters, Bert (ed.). The lexicon-encyclopedia interface. Amsterdam : Elsevier Publishing, 2000. 97-114.

Inchaurralde, Carlos ; Vázquez, Ignacio (eds.) (2000). Una introducción cognitiva al lenguaje y la lingüística. Zaragoza : Mira, 2000.

Keatley, C. ; De Gelder, B. (1992). The bilingual primed lexical decision task: Cross-language priming disappears with speeded responses. // European Journal of Cognitive Psychology. $4: 4$ (1992) 273-292.

Keatley, C. ; Spinks, J. ; De Gelder, B. (1994). Asymmetrical cross-language priming effects. // Memory and Cognition. 22 : 1 (1994) 70-84.

Lakoff, George. (1987). Cognitive models and prototype theory. // Neisser, Ulric (ed.). Concepts and conceptual development. Ecological and intellectual factors in categorization. Cambridge : Cambridge University Press, 1987. 63-100.

Langacker, Ronald W. (1987). Foundations of cognitive grammar : vol. 1. Theoretical prerequisites. Stanford : Stanford University Press, 1987.

Long, M. (1985). A role for instruction in second language acquisition: task-based language teaching. // Hyltenstam, K. ; Pienemann, M. (eds.). Modelling and assessing second language acquisition. Clevedon, Avon : Multilingual Matters, 1985.

Long, M. ; Crookes, G. (1992). Three approaches to task-based syllabus design. // TESOL Quarterly. 26 (1992) 27-56.

Marslen-Wilson, William D. (1987). Functional parallelism in spoken word recognition. // Cognition. 25 (1987) 71-102.

Scire. 8: 1 (en.-jun. 2002) 41-54. 
McClelland, J. ; Rumelhart, D. (1981). An interactive-activation model of context effects in letter perception, part 1: An account of basic findings. // Psychological Review. 88 (1981) 375-405.

Morton, John (1979). Word recognition. // Morton, John ; Marshall, John C. (eds.). Psycholinguistics series: vol. 2: Structures and processes. London : Elek, 1979. 107-156.

Nelson, Katherine (1985). Making sense: The acquisition of shared meaning. New York : Academic Press, 1985.

Osgood, C. (1976). Focus on meaning: Volume I: explorations in semantic space. The Hague : Mouton, 1976.

Patterson, Karalyn E. ; Shewell, Christina (1987). Speak and spell. Dissociations and word-class effect. // Coltheart, Max ; Sartori, Giuseppe ; Job, Remo (eds.). The cognitive neuropsychology of language. Hillsdale : Lawrence Erlbaum, 1987. 273-294.

Prabhu, N. S. (1987). Second language pedagogy. Oxford : Oxford University Press, 1987.

Putnam, H. (1975). Mind, language and reality: philosophical papers II. Cambridge : Cambridge University Press, 1975.

Reddy, Michael J. (1979). The conduit metaphor. A case of frame conflict in our language about language. // Ortony, Andrew (ed.). Metaphor and thought. London : Cambridge University Press, 1979. 284-324.

Rosch, Eleanor (1973). Natural categories. // Cognitive Psychology. 4 (1973) 328-350.

Rosch, Eleanor (1975). Cognitive representations of semantic categories. // Journal of Experimental Psychology: General 104, 1975. 192-233.

Rosch, Eleanor (1977). Human categorization. // Warren, N. (ed.). Studies in cross-cultural psychology. London : Academic Press, 1977.

Rosch, Eleanor (1978). Principles of categorization. // Rosch, E. ; Lloyd, B. B. (eds.). Cognition and categorization. Hillsdale, N.J.: Lawrence Erlbaum, 1978.

Rosch, Eleanor (1981). Prototype classification and logical classification: The two systems. // Scholnick, E. (ed.). New trends in cognitive representation: challenges to piaget's theory. Hillsdale, N.J. : Lawrence Erlbaum, 1981.

Rosch, E. ; Lloyd, B. B. (eds.) (1978). Cognition and categorization. Hillsdale, N.J. : Lawrence Erlbaum, 1978.

Rosch, E. ; Mervis, C. (1975). Family resemblances: Studies in the internal structure of categories. // Cognitive Psychology. 7 (1975) 573-605.

Sanford, Anthony J. ; Garrod, Simon C. (1981). Understanding written language. New York : John Wiley, 1981.

Taylor, John (1989). Linguistic categorization. Prototypes in linguistic theory. Oxford : Clarendon, 1989.

Tulving, Endel (1979). Relation between encoding specificity and levels of processing. // Cermak, Laird S. ; Craik, Fergus I. M. (eds.). Levels of processing in human memory. Hillsdale : Lawrence Erlbaum, 1979. 405-428.

Wierzbicka, Anna (1997). Understanding cultures through their key words. English, russian, polish, german, and japanese. Oxford : Oxford University Press, 1997. 